Drop spreading and penetration into pre-wetted powders

\author{
J.O. Marston \\ J.E. Sprittles \\ Y. Zhu \\ E.Q. Li \\ I.U. Vakarelski \\ S.T. Thoroddsen
}





\title{
Drop spreading and penetration into pre-wetted powders
}

\author{
J.O. Marston ${ }^{1, *}$, J.E. Sprittles ${ }^{2}$, Y. Zhu ${ }^{1}$, E.Q. Li ${ }^{1}$, \\ I.U. Vakarelski ${ }^{1,3} \&$ S.T. Thoroddsen ${ }^{1,3}$ \\ ${ }^{1}$ Division of Physical Sciences and Engineering, King Abdullah University of \\ Science and Technology, Thuwal, 23955-6900, Saudi Arabia. \\ 2 Mathematical Institute, University of Oxford, 24-29 St Giles, \\ Oxford OX1 3LB, UK. \\ ${ }^{3}$ Clean Combustion Research Centre, King Abdullah University of Science and \\ Technology, Thuwal, 23955-6900, Saudi Arabia.
}

\begin{abstract}
We present results from an experimental study of the impact of liquid drops onto powder beds which are pre-wetted with the impacting liquid. Using high-speed video imaging, we study both the dynamics of the initial spreading regime and drainage times once the drop has reached its maximum spread on the surface. During the initial spreading stage, we compare our experimental data to a previously developed model which incorporates imbibition into the spreading dynamics and observe reasonable agreement. We find that the maximum spread is a strong function of the moisture content in the powder bed and that the total time from impact to complete drainage is always shorter than that for dry powder. Our results indicate that there is an optimum moisture content (or saturation) which leads to the fastest penetration. We use simple scaling arguments which also identify an optimum moisture content for fastest penetration, which agrees very well with the experimental
\end{abstract}


result.

Key words: Drop impact, Maximum deformation, drainage, high-speed imaging PACS:

\section{Introduction}

In wet granulation, liquid is added to powder with the aim of forming granules, which have more desirable handling and flow properties for tablet formation. It is known that the first key step in this process, i.e. the wetting and nucleation stage, is crucial to the success of the whole granulation process [1]. Thus it is important to understand the dynamics of this wetting process. As such, the topic of drop impact onto powder surfaces has received renewed interest $[2-7]$ in order to study phenomena such as the temporal evolution of the spreading phase, the maximum deformation of the drop and the resulting crater morphology.

When the drop wets the powder, the ensuing imbibition into the bed can broadly be characterised by two limiting cases; The first is known as the constant drawing area (CDA) where the contact line is pinned; the second is known as the decreasing drawing area (DDA) where the contact angle remains constant but the contact line retracts. The corresponding times for penetration in these two cases are given by $[8,9]$ as

$$
\begin{gathered}
t_{C D A}=1.35 \frac{V_{0}^{2 / 3}}{\epsilon^{2} R_{\text {pore }}} \frac{\mu}{\sigma \cos \theta_{p}} \\
t_{D D A}=9 t_{C D A}
\end{gathered}
$$

where $V_{0}$ is the initial drop volume, $\mu$ is the liquid dynamic viscosity, $\epsilon$ is the 
powder bed porosity, $\sigma$ is the liquid surface tension and $\theta_{p}$ is the apparent contact angle. $R_{\text {pore }}=2 \epsilon /\left((1-\epsilon) S_{0} \rho_{s}\right)$ is the effective size of the pores in the bed, where $S_{0}$ is the particle specific surface area and $\rho_{s}$ is the packing density of the pores.

Hapgood et al. [9], however, provide a modified expression for the effective pore size based on particle shape and effective porosity as

$$
R_{e f f}=\frac{\Phi d_{3,2}}{3} \frac{\epsilon_{e f f}}{\left(1-\epsilon_{e f f}\right)}
$$

where $\Phi$ is a shape factor, $d_{3,2}$ is the surface mean particle diameter, and $\epsilon_{e f f}=\epsilon_{t a p}\left(1-\epsilon+\epsilon_{t a p}\right)$. This modification, when substituted into equation (1), was found to give a better fit to experimental data for several powders where the penetration times spanned three orders of magnitude.

When the powder surface is non-wettable, the drop may rebound from the powder surface with a partial or full coating of powder from the bed $[2,3]$, thus dramatically increasing the lifetime of the drop on the powder surface. In particular, the work of Hapgood and co-workers [10-13] has shown the possibility of using hydrophobic powder in the production of novel, hollow granules from structures known as liquid marbles $[3,14,15]$.

Hapgood et al. [16] studied the motion and penetration of drops which are released onto a powder surface at a controlled separation distance from a prewetted area on the powder bed (i.e. the impact site of a previously released drop). They found that the probability of forming two nuclei is dependent not only on the separation distance, but also the drop penetration time. In addition, Charles-Williams et al. [17] have studied the penetration of drops 
into both dry and pre-wetted powders. In their experiments, the pre-wetted powder was defined as the impact site of a previous drop, thus the moisture content was not a control parameter and the penetration times for the prewetted powder was always longer than that for dry powder.

In this study, as with those of [16,17], we are motivated by the fact that during a true wet granulation process, the binder drops will impact onto not only dry, but partially wet or saturated powders. We thus extend the studies of $[16,17]$ by examining the evolution of a drop after impact onto a pre-wetted powder, where we systematically vary the moisture content in the bed.

\section{Materials and methods}

The experimental setup (see Figure 1) consists of a small cylindrical container, filled with fine glass beads. The beads have a mean diameter $d_{4,3}=31 \mu \mathrm{m}$ $\left(d_{10}=19 \mu \mathrm{m}, d_{90}=46 \mu \mathrm{m}\right)$ and circularity $C=\frac{4 \pi A}{P^{2}}=0.96$, as determined by particle size and shape analysis on a combination of a Malvern Mastersizer and Malvern Morphologi G3. Upon substituting $C$ for $\Phi$ in equation (3), we estimate the effective pore radius to be $R_{e f f} \approx 4.5 \mu \mathrm{m}$. The contact angle was determined using the mass-based capillary rise method [18,19] according to the following equation:

$$
m^{2}=c \frac{\rho^{2} \sigma \cos \theta}{\mu} t
$$

where $m$ is the mass of liquid in the capillary at time, $t, \rho$ is the liquid density and $c$ is a material constant. The experiments were performed on a force tensiometer (Sigma 701, KSV Instruments) and $c$ was determined using per- 
fluorohexane $\left(\mathrm{C}_{6} \mathrm{~F}_{14}\right)$, a wetting fluid with very low surface tension, $\sigma=11.9$ $\mathrm{mN} / \mathrm{m}$. The resulting contact angle of water on the beads was $\theta=61^{\circ} \pm 3^{\circ}$.

For dry beds, the beads are simply poured into the container, compressed slightly and scraped level. For wet powders, a specific mass of beads $\left(m_{\text {beads }}\right)$ was first mixed with a specific mass of water $\left(m_{l i q}\right)$ in order to yield a known moisture content, given by

$$
M=\frac{m_{\text {liq }}}{m_{\text {beads }}}
$$

We use set moisture contents of $M=2.5,5,10,15,20$ and $25 \%$, yielding bulk densities $\rho_{\text {bulk }}=1.44,1.49,1.61,1.68,1.71$ and $1.72 \mathrm{~g} / \mathrm{cm}^{3}$ and approximate saturations of $S=8.3,17,37,59,75$ and $89 \%$ based on the preparation methods used herein.

We then release a drop of pure water from heights $h_{r} \approx 1 \mathrm{~mm}$ up to $80 \mathrm{~cm}$. The drops are dispensed from a custom-made glass capillary, whose nozzle size can be modified to alter the drop diameter, however, to isolate the influence of the moisture content of the powder, we chose a single drop size of $D_{0}=2.1$ $\mathrm{mm}$, resulting in a drop volume of $V_{0}=4.85 \mu \mathrm{L}$ in all trials. The drops thus impact vertically with speed $u_{i} \approx \sqrt{2 g h_{r}}=0.14-3.9 \mathrm{~m} / \mathrm{s}$, which is measured directly from the video sequences. The main dimensionless parameter used to characterise the impact is the Weber number, $W e=\rho D_{0} u_{i}^{2} / \sigma$, which varies from $\sim 0.5$ to 440 . The Bond number for this study, $B o=\rho g D_{0}^{2} / \sigma=0.59$ and the Ohnesorge number, $O h=\mu / \sqrt{\rho \sigma D_{0}}=2.6 \times 10^{-3}$. Given that the drop diameters, $2.1 \mathrm{~mm}$, are smaller than the capillary length, $a=\sqrt{\sigma /(\rho g)}=2.7$ $\mathrm{mm}$, we expect the drops to be spherical at impact, which is verified from the image sequence except for the lowest release heights where some minor 
oscillations occur due to the pinch-off.

The impact, spreading and drainage are all captured in a single video sequence by a high-speed video camera (Phantom V1610, Vision Research) equipped with a $1 \times$ objective lens, which yields effective pixel sizes of $27 \mu \mathrm{m} / \mathrm{px}$. Frames rates up to $10,000 \mathrm{fps}$ were used.

All trials were conducted in an air-conditioned laboratory with ambient temperature of $21-22{ }^{\circ} \mathrm{C}$ and relative humidity, $\mathrm{RH}=50-55 \%$. 


\section{Qualitative features}

Figure 2 shows three series of images from high-speed video sequences for the spreading and drainage of a water drop in (a) dry powder, (b) partially wetted powder with $M=2.5 \%$ and (c) almost saturated powder with $M=25 \%$. In these realisations, the drop was released from just above the surface $\left(h_{r}=1.2\right.$ $\mathrm{mm}, u_{i}=0.15 \mathrm{~m} / \mathrm{s}$ ). The spreading phase in (a) appears to be characteristic of a non-wetting target surface whilst (b) and (c) clearly exhibit characteristics of a drop spreading on a hydrophilic surface [20-22]. The total drainage time, taken from impact until all the liquid has been imbibed, is 211, 34 and 128 ms respectively.

At higher impact speed with $u_{i}=2.2 \mathrm{~m} / \mathrm{s}$, Figure 3 shows the equivalent sequences for drops impacting the same target surfaces as in Figure 2. Here, in contrast to the low-impact speed, the dynamics appear both qualitatively and, to some extent, quantitatively similar for the first 4 panels in each series. However, we observe clear differences at later times. For instance, the drop on dry powder generates a small crater and a granule formation. For the two wet powders, we also observe a slight difference in the maximum deformation and again, a three-fold difference in the total drainage times.

At a qualitative level, the moisture content of the powder does not dramatically influence the very initial spreading dynamics. However, the maximum spread diameter and the drainage process appear to be significantly influenced by the moisture content. 


\section{Spreading}

\subsection{Spread rates}

For wet powders, the spreading, as shown by Figures 2 and 3, appears to be characterisitc of a hydrophilic surface and there may also be some penetration during the spreading phase, so that one would expect the maximum spreading to be influenced by not only the drop impact speed (i.e. kinetic energy), but also the moisture content of the powder. Figures 3(b) and (c) would certainly indicate that this is the case. Figures $4(\mathrm{a})-(\mathrm{d})$ plot the spread diameter as a function of time from impact for a range of impact speeds and moisture contents. Each data set is plotted only up to the maximum spread, after which drainage begins. Note that for all moisture contents and impact speeds, the maximum spread is reached within $10 \mathrm{~ms}$ from impact, thus highlighting the rapidity of the dynamics. The transition from low-impact speed to highimpact speed is qualitatively similar for all moisture contents shown here and the maximum spread diameter appears to increase monotonically with impact speed.

\subsection{Comparison with theory}

A continuum model for the impact, spreading and imbibition of a drop into porous powder bed requires the solution of the Navier-Stokes equations in the drop, coupled to the Darcy equations which describe flow through the porous medium. These bulk equations must be combined with boundary conditions which, in particular, include a description of the dynamic contact angle which the drop's free surface makes with the porous medium. The formulation of 
this problem is described in detail in [25], and will be the subject of future work; however, as a first step, the experimental results of Figure 4 will now be compared to the model presented in [26], where a number of simplifying assumptions allow for the evolution of the drop to be obtained without resorting to complex computational calculations.

The model presented in [26], for what is known as the 'increasing drawing area' (IDA) regime, has been analysed in detail in [27], where a step-by-step guide to the assumptions of the model, its derivation and numerical implementation have been provided, so that here we shall just briefly recapitulate the main details. It is assumed that throughout the spreading process the drops remains a spherical cap which is characterized by the contact angle at which the free surface meets the powder bed. The relationship between the volume of the drop $V_{d}(t)$, the wetted radius $r=d / 2$ and the dynamic contact angle $\theta_{d}$ is then given by

$$
V_{d}=\xi\left(\theta_{d}\right) r^{3}, \quad \text { where } \quad \xi\left(\theta_{d}\right)=\frac{\pi}{3} \frac{\left(1-\cos \theta_{d}\right)\left(\cos \theta_{d}+2\right)}{\sin \theta_{d}\left(\cos \theta_{d}+1\right)}
$$

A key assumption in the model of [26] is that the evolution of the dynamic contact angle can be obtained by using a formula $[28,29]$ which was derived for spreading on impermeable substrates:

$$
\theta_{d}=F\left(\frac{\zeta \dot{r}}{\sigma}, \theta_{e}\right), \quad \text { where } \quad F=\cos ^{-1}\left(\cos \theta_{e}-\frac{\zeta \dot{r}}{\sigma}\right)
$$

where $\theta_{e}$ is the 'equilibrium' contact angle, $\zeta$ is the 'coefficient of wetting-line friction' and $\dot{r}$ is the contact line speed. This is of course not strictly valid, as on a porous bed there is no such thing as an equilibrium contact angle, as 
a drop placed on such a substrate will seep into it. However, as a first step we simply use the formula suggested and fit the two parameters $\theta_{e}$ and $\zeta$, in order to at least capture some of the interplay between the spreading of the drop and imbibition.

Imbibition into the porous matrix is assumed analogous to the imbibition into an array of vertical capillaries of radius $R_{t}$, so that no radial flow occurs, and, after some manipulation, this gives the volume of liquid inside the porous $\operatorname{matrix} V_{p}(t)$ as

$$
V_{p}(t)=\pi \epsilon \sqrt{\frac{R_{t} \sigma \cos \theta_{p}}{8 \mu}} \int_{0}^{t} \frac{r^{2}(t-\tau)}{\sqrt{\tau}} d \tau .
$$

Noting that the total volume $V_{0}=V_{d}(t)+V_{p}(t)$ is conserved, equations (5) and (7) produce an integro-differential equation for the drop radius $r$ as a function of time

$$
\xi\left(\theta_{d}\right) r^{3}+\pi \epsilon \sqrt{\frac{R_{t} \sigma \cos \theta_{p}}{8 \mu}} \int_{0}^{t} \frac{r^{2}(t-\tau)}{\sqrt{\tau}} d \tau=V_{0}
$$

where $\theta_{d}$ is given by (6). Equation (8) has been solved using the numerical methods described in [27].

Once non-dimensionalised, this model produces three parameters which can be used to fit the data: the equilibrium contact angle $\theta_{e}$, the dimensionless coefficient of wetting line friction $\bar{\zeta}=\zeta / \mu$ and a parameter $\lambda=\epsilon \sqrt{\frac{2 R_{t} \cos \theta_{p}}{D_{0}}}$ which characterises the ability of the porous medium to draw liquid from the drop.

The results of this IDA model are shown in Figure 5 for the initial spreading phase of the liquid drop across the powder bed for the lowest impact speed, where the model is most likely to be valid, at four different moisture contents. 
In all cases we have seen that $\lambda \approx 0$, so that during this initial phase there is negligible imbibition of liquid into the powder bed, i.e. the time scale for imbibition is much longer than that of the initial spread which takes only a few milliseconds. This is consistent with experimental observations which show that indeed, very little liquid is lost in the time it takes for the drop to reach its maximum diameter. The remaining fitted parameters are then given by:

\begin{tabular}{|c|c|c|}
\hline$M$ & $\bar{\zeta}$ & $\theta_{e}$ \\
\hline $2.5 \%$ & 202 & $6.1^{\circ}$ \\
$5 \%$ & 259 & $26.2^{\circ}$ \\
$10 \%$ & 210 & $8.2^{\circ}$ \\
$20 \%$ & 169 & $0^{\circ}$ \\
\hline
\end{tabular}

As a comparison, the red dotted line in Figure 5 shows the effect of forcing some imbibition for the $M=10 \%$ case, by taking $\lambda=0.01$, and finding that the best fit was then obtained for $\bar{\zeta}=193$ and $\theta_{e}=0^{\circ}$. As can be seen graphically, the agreement between experiment and theory is clearly worse, which confirms our initial assumption that $\lambda \approx 0$ for the IDA phase.

Care must be taken in extracting conclusions from this data because, as can be seen from the free surface shapes shown in Figure 2, assumptions such as that of sphericity of the drop are clearly violated. However, broadly, as typical values for the $\bar{\zeta}$ on smooth impermeable substrates are around $5-50$, one can say that, as also found in $[25,27]$, a reasonable fit can be obtained when 
the powder beds are characterised as wettable high-friction surfaces. In other words, the effect of moisture enhances the wettability of the porous bed, as compared to a dry porous medium, but the inherent roughness of the surface on which the drop spreads slows down the drop compared to one spreading on a smooth impermeable substrate.

\subsection{Maximum spread}

For drops impacting onto dry solid surfaces, the maximum spread has been assessed by several authors, generally taking the approach of balancing impact kinetic energy with surface energy or inertia with surface tension, leading to scalings in terms of the Weber number of $d_{\max } / D_{0} \sim W e^{1 / 2}$ or $W e^{1 / 4}$ respectively [23,24]. For impact onto dry, wettable powders, Marston et al. [2] found that $d_{\max } / D_{0} \sim W e^{1 / 5}$ for low-surface tension or low-viscosity liquids, which was later confirmed by Nefzaoui \& Skurtys [7], whilst for water drops impacting onto hydrophopbic powders [3], the scaling changes dramatically with $d_{\max } / D_{0} \sim W e^{2 / 5}$.

Figure 6(a) plots the maximum spread diameter as a function of impact speed for different moisture contents assessed in this study, including dry powder (shown by solid black circles) for reference. For low-impact speeds up to $u_{i} \approx$ $2 \mathrm{~m} / \mathrm{s}$, it is clear that the maximum spread increases monotonically with moisture content. For higher impact speeds, however, the data becomes more scattered, which may be due to increasing competition between inertia-driven spreading and penetration into the pre-wetted void structures.

The data from Figure 6(a) is replotted in Figure 6(b) where $d_{\max }$ has been normalised with respect to the initial drop diameter, $D_{0}$, and the impact speed 
with respect to surface tension, in terms of the impact Weber number. Here, we have also included the limiting values of the slopes obtained from fitting the data to $d_{\max } / D_{0} \sim W e^{\alpha}$, where $\alpha \approx 1 / 5$ for dry powder and $\alpha \approx 1 / 10$ for pre-wetted powder at high moisture contents. The dependence of $\alpha$ on $M$ is shown in Figure 6(c). Note that in this fitting procedure, we have excluded the low-impact speed data since we are focusing on regime in which the spreading is driven by inertia. For pre-wetted powders, the drop spreads easily across the surface due to the presence of liquid, not just in the pores, but on the surface of the top layer of grains, which ultimately results in significantly reduced contact line friction, leading to increased spread diameters. However, the spreading will be arrested not just by surface tension, as is the case for non-porous or hydrophobic substrates, but also by penetration into the powder bed. As the moisture content increases, however, (e.g. for $M=25 \%$ ), the pores become more saturated and thus we expect a weaker influence of penetration during the spreading phase, which is observed in Figure 6(c) for $M=25 \%$. 


\section{Drainage}

\subsection{Drainage type}

In Figure 7, we present three example sequences of the drainage process into (a) dry and $(b, c)$ pre-wetted powders. The scale bars at the top of each sequence indicate the maximum contact diameter from which the drainage starts. Note that for the pre-wetted powders, this is precisely the same as $d_{\text {max }}$, obtained during the spreading phase, but not for dry powders since the drops will often first rebound before penetration commences.

Figure $7(d)$ shows the diameter of the apparent contact area, $d_{a p p}$, versus time for the three examples in Figure 7 (a), (b) and (c). The final data point for the dry powder is the last measurement that could be taken due to the crater obscuring the camera view.

The absolute times for complete drainage, when measured from the moment of impact, vary significantly with $t_{\text {drain }} \approx 211,34$ and $128 \mathrm{~ms}$ (for (a), (b) and (c) respectively). Note that for dry powder, complete drainage was only observed for the lowest impact speeds (i.e. $u_{i}=0.15 \mathrm{~m} / \mathrm{s}$ ) when the drop was released just above the powder surface. For all other impact speeds studied herein, a granule is formed (e.g. Fig 3(a)). The total time from impact to either drainage or granule formation for dry powders is independent of impact speed with mean time, $\bar{t} \approx 247 \mathrm{~ms}$ and standard deviation, std $=30 \mathrm{~ms}$.

In the case of the dry powder, in Figure 7(a), we observe that the apparent contact diameter is roughly constant during the penetration, thus in agreement with the experiments of Hapgood et al. [9] for water on glass beads, it appears 
that the drainage occurs over a constant drawing area (CDA).

In contrast, for the pre-wetted powders, as in Figures 7 (b) and (c), the apparent contact diameter is clearly time-dependent and diminishes as time progresses towards complete drainage. From these experimental observations of the apparent contact diameter alone it is difficult to say whether the drainage type is that of decreasing drawing area (DDA) or whether it is a modified version of the CDA-type, where a thin remnant film remains on the powder surface. This modified CDA-type drainage was described in Denesuk et al. [14] and a schematic of this process is shown in Figure 8(a). Intuitively, it is reasonable to expect that this modified CDA-type drainage occurs given that the powders are pre-wet and we present evidence that this is indeed the case.

Following Denesuk et al. [8], in the modified CDA regime, where a thin remnant film persists on the powder surface, we can express the time-dependent apparent contact diameter as:

$$
d_{\text {app }}(t)=d_{\max }\left(1-\sqrt{\frac{t}{t_{C D A}}}\right)^{1 / 3}
$$

where both $d_{\max }$ and $t_{C D A}$ are easily measured from the video sequences. In contrast, for a true DDA-type drainage, the corresponding apparent contact diameter is given simply by:

$$
d_{\text {app }}(t)=d_{\max }\left(1-\sqrt{\frac{t}{t_{D D A}}}\right) .
$$

These two equations then allow us to quantitatively assess which type of drainage occurs in our experiments. Figure 8(b) plots $d_{a p p}(t)$ from equations (4) and (5) based on an arbitrary initial contact diameter. These curves show 
that for DDA, the magnitude of the slope decreases with time, whereas for the modified CDA, the slope initially decreases but then increases more rapidly as the drainage progresses. The latter provides a better qualitative description of the drainage for the pre-wet powders, as shown by Figure 9 for 3 different realisations with $M=2.5 \%$. The predicted evolution of $d_{\text {app }}$ from the DDA limit is clearly qualitatively incorrect, whereas the predicted evolution from the modified CDA-type drainage does correctly predict the increasing rate of drainage as time progresses, which is seen in the experiments. The overall quantitative agreement deteriorates with increasing impact speed or, equivalently, initial contact diameter. From Figures 10 (a)-(d), we also find better agreement for lower moisture contents, where we plot the evolution of the apparent contact diameter for different values of $M$ and $u_{i}$. For both Figures 9 and 10, the values of $t_{C D A}$ are taken as the time from maximum contact diameter to complete drainage, which is the true period of time over which the drainage process take places. Note that this simple model was derived from arguments based on an ideal porous surface with parallel cyndrical pores of equal diameter which, as noted by Hapgood et al. [9], cannot strictly be applied to true powder beds as they contain complex heterogenous void structures. Nonetheless, the characteristic features of the drainage observed in the experiments appear to support the modified CDA-type drainage model.

\subsection{Drainage time}

The total time for the drop to completely drain into the powder bed, taken from the moment of impact, is presented in Figure 11. Here we can clearly observe the independence of $t_{d}$ on $u_{i}$ for the dry powder (shown by solid blue data points) with a mean drainage time of $247 \mathrm{~ms}$. For the pre-wetted 
powders with $M<25 \%$, we find that $t_{d}$ can reduce by a factor of 5 or more with $t_{d}=30-138 \mathrm{~ms}$ for the lowest impact speed and $t_{d}=6-43 \mathrm{~ms}$ for the highest impact speeds. We note that for $M=25 \%$, there is a sudden increase in $t_{d}$ for $u_{i}>2.5 \mathrm{~m} / \mathrm{s}$, which is highlighted by the dotted black line. This particular observation is not yet understood, however, it is likely to be due to the complex competition between increasing drop impact kinetic energy, powder compression and initial saturation. In general, we find that the shortest drainage times are achieved with $M=5 \%$, shown by the red data points. This is further emphasized in Figure 12, where we plot $t_{d}$ versus $M$ for select impact speeds. Each data set shows a local minimum occurring at $M=5 \%$, thus indicating that there is an optimum initial moisture content or saturation that induces the fastest penetration.

To understand this optimum moisture content, we revert to the original expression for drainage time proposed by Denusek et al. [8], which can be expressed in the following form:

$$
t_{C D A}=\frac{A R_{e f f}^{3}}{\cos \left(\theta_{p}\right) k^{2}}
$$

where $A$ is a constant depending on several material parameters, $R_{\text {eff }}$ is the effective pore radius, $\cos \left(\theta_{p}\right)$ quantifies the pore wettability, and $k$ is the permeability. We now seek to express how these variables behave with increasing moisture content. If we let $\bar{M}=M / M_{\max }$, where $M$ is the moisture content and $M_{m a x}$ represents the moisture content at saturation, then $\bar{M}=0$ for dry powder and $\bar{M}=1$ for saturated powder. The permeability, $k \sim R_{\text {eff }}^{2}$, so that

$$
k^{2} / R_{e f f}^{3} \sim R_{e f f} \equiv R_{d r y}(1-\bar{M})
$$


where $R_{d r y}=R_{e f f}(M=0)$. We also know that the contact angle on the dry bed, $\theta_{p} \approx 60^{\circ}$, so we can write

$$
\theta_{p}(M)=60(1-\bar{M})
$$

Substituting equations (8) and (9) into (7), we then have

$$
t_{C D A}=\frac{A}{\cos (60[1-\bar{M}])[1-\bar{M}]} .
$$

This expression for $t_{C D A}$ has a minimum at $\bar{M}=0.18$, which for our experiments corresponds to $M=5.4 \%$. Whilst this is in good quantitative agreement with the experimental observations, we emphasize that we have again used some overly simplistic assumptions of how parameters in equation (11) depend on moisture content, which will be addressed in future work. Nonetheless, the observation of an optimum initial moisture content may prove useful for applications where fast liquid penetration or drainage is desirable or simply for extending the regime maps for granule formation (e.g. Emady et al. [30]). Using humidified air has already been used at the pilot-scale to achieve binderless granulation $[31,32]$ and could also be used to pre-fluidise powder beds to increase the initial bed moisture content prior to binder spray addition that may then result in faster wetting of the powder for granulation. 


\section{Concluding remarks}

We have performed an experimental study of the impact of water drops onto pre-wetted powders with a range of moisture contents. During the very initial spreading regime, the moisture content has little effect and the spreading appears both qualitatively and quantitatively similar for both low $(\sim 2.5-5 \%)$ and high $(\sim 20-25 \%)$ moisture contents. In contrast however, the maximum spread and subsequent drainage of the drop into the powder are significantly influenced by the moisture content and impact speed.

For low-impact speed, we have compared our experimental data to the model of Clarke et al. [26], which indicates that there is little imbibition during the initial spreading phase. The fit to the theory, however, relies upon several overly simplistic assumptions and the development of a full theoretical treatment of this problem which will extend the work of [25] is the focus of on-going work.

The maximum spread of the drops can be expressed in terms of the impact Weber number as $d_{\max } / D_{0} \sim W e^{\alpha}$, but the scaling exponent, $\alpha$, becomes a function of the moisture content, $M$.

For impact onto dry powder, the drop only drains for low impact speeds, whilst at higher impact speeds we observe the formation of granule nuclei. In stark contrast, for wet powders at all moisture contents and drop impact speeds, we observe full drainage of the drop into the powder. Our observations of the apparent contact diameter indicate that the drainage type is a modified CDA-type, proposed by Denesuk et al. [8]. Granules are never observed for pre-wetted powders. We have also not observed any splashing during impact onto pre-wetted powders. 
The total time from impact to complete drainage spans two orders of magnitude and is a strong function of both the moisture content in the powder bed and the drop impact speed or, equivalently, the maximum spread diameter. In contrast to Charles-Williams et al. [17], we find that the penetration times for pre-wetted powder are always shorter than for dry powders and that there is an optimum saturation that induces the fastest penetration. Our experiments indicate that this value is achieved with $M \approx 5 \%$ or $S \approx 17 \%$, which is supported simple arguments based on the effective pore radius, contact angle and permeability of the powder bed. This value may vary depending on the size and shape of the grains and further investigation into the drainage of drops onto pre-wetted powders should include both of these parameters. The regime maps for granule formation also need to be re-addressed in light of this finding.

\section{Acknowledgement}

This work was partially supported by an Academic Excellence Alliance grant (7000000028) awarded by the KAUST Office of Competitive Research Funds.

\section{References}

[1] Iveson, S.M., Lister, J.D., Hapgood, K. \& Ennis, B.J., Nucleation, growth and breakage phenomena in agitated wet granulation processes: a review Powder Tech. 117 (2001) 3-39.

[2] Marston, J.O., Thoroddsen, S.T., Ng, W.K. \& Tan, R.B.H., Experimental study of liquid drop impact onto a powder surface Powder Tech. 203 (2010) 223-236. 
[3] Marston, J.O., Zhu, Y., Vakarelski, I.U. \& Thoroddsen, S.T., Deformed liquid marbles: Freezing drop oscillations with powder Powder Tech. 228, 424-428.

[4] Emady, H.N., Kayrak-Talay, D., Schwerin, W. \& Lister, J.D. Granule formation mechanisms and morphology from single drop impact on powder beds Powder Tech. 212 (2011) 69-79.

[5] Katsuragi, H., Morphology scaling of drop impact onto a granular layer Phys. Rev. Lett. 104 (2011) 218001.

[6] Katsuragi, H., Length and time scales of a liquid drop impact and penetration into a granular layer J. Fluid Mech. 675 (2011) 552-573.

[7] Nefzaoui, E. \& Skurtys, O.R., Impact of a liquid drop on a granular-medium: Inertia, viscosity and surface tension effects on the drop deformation Exp. Thermal \& Fluid Sci. doi: 10.1016/j.expthermflusci.2012.03.007 (2012).

[8] Denusek, M., Smith, G.L., Zelinski, B.J.J., Kreidl, N.J. \& Uhlmann, D.R., Capillary penetration of liquid droplets into porous materials J. Colloid 8 Interface Sci. 158 (1993) 114-120.

[9] Hapgood, K.P., Lister, J.D., Biggs, S.R. \& Howes, T., Drop penetration into porous powder beds J. Colloid ES Interface Sci. 253 (2002) 353-366.

[10] Eshtiaghi, N., Liu, J.S. \& Hapgood, K.P., Formation of hollow granules from liquid marbles: Small scale experiments Powder Tech. 197 (2010) 184-195.

[11] Hapgood, K.P. \& Khanmohammadi, B., Granulation of hydrophobic powder Powder Tech. 189 (2009) 253-262.

[12] Eshtiaghi, N., Liu, J.S., Shen, W. \& Hapgood, K.P., Liquid marble formation: Spreading coefficients or kinetic energy? Powder Tech. 196 (2009) 126-132.

[13] Estiaghi, N. \& Hapgood, K.P., A quantitative framework for the formation of liquid marbles and hollow granules from hydrophobic powders Powder Tech. 223 (2012) 65-76. 
[14] McHale, G. \& Newton, M.I., Liquid Marbles: principles and applications Soft Matter 7 (2011) 5473-5481.

[15] Aussillous, P. \& Quere, D., Properties of liquid marbles Proc. Roy. Soc. A 462 (2006) 973-999.

[16] Hapgood, K.P., Nguyen, T.H., Hauw, S., Iveson, S.M. \& Shen, W., Rewetting effects and droplet motion on partially wetted powder surfaces AIChE J. 55(6) (2009) 1402-1415.

[17] Charles-Williams, H.R., Wengeler, R., Flore, K., Feise, H., Hounslow, M.J. \& Salman, A.D., Granule nucleation and growth: Competing drop spreading and infiltration processes Powder Tech. 206 (2011) 63-71.

[18] Nowak, E., Combes, C., Stitt, E.H. \& Pacek, A.W., A comparison of contact angle measurement techniques applied to highly porous catalyst supports Powder Tech. 233, (2012) 52-64.

[19] Siebold, A., Walliser, A., Nardin, M., Oppliger, M. \& Schultz, J., Capillary rise for thermodynamic characterization of solid particle surface J. Colloid $\mathcal{E}$ Interface Sci. 186, (1997) 60-70.

[20] Ding, H., Li, E.Q., Zhang, F.H., Sui, Y., Spelt, P.D.M. \& Thoroddsen, S.T., Propagation of capillary waves and ejection of small droplets in rapid droplet spreading J. Fluid Mech. 697 (2012) 92-114.

[21] Rioboo, R., Adao, M.H., Voue, M. \& De Coninck, J., Experimental evidence of liquid drop break-up in complete wetting experiments J. Mater. Sci. 41 (2006) 5068-5080.

[22] Biance, A-L., Clanet, C. \& Quere, D., First steps in the spreading of a liquid droplet Phys Rev. E 69 (2004) 016301.

[23] Renardy, Y., Popinet, S., Duchemin, L., Renardy, M., Zaleski, S., Josserand, C., Drumright-Clarke, M.A., Richard, D., Clanet, C. \& Quere, D., Pyramidal 
and toroidal water drops after impact on a solid surface J. Fluid Mech. $\mathbf{4 8 4}$ (2003) 69-83

[24] Clanet, C., Beguin, D., Richard, D. \& Quere, D., Maximal deformation of an impacting drop J. Fluid Mech. 517 (2004) 199-208.

[25] Shikhmurzaev, Y.D. \& Sprittles, J.E., Dynamic contact angle of a liquid spreading on an unsaturated wettable porous substrate J. Fluid Mech. 715, (2013) 273-282.

[26] Clarke, A., Blake, T.D., Carruthers, K. \& Woodward, A., Spreading and imbibition of liquid droplets on porous surfaces Langmuir 18, (2002) 2980-2984.

[27] Hilpert, M. \& Ben-David, A., Infiltration of liquid droplets into porous media: Effect of dynamic contact angle and contact angle hysteresis Int. J. Multiphase Flow 35, (2009) 205-218.

[28] Blake, T.D. \& Haynes, J.M., Kinetics of liquid/liquid displacement J. Colloid E Interface Sci. 30, (1969) 421-423.

[29] Blake, T.D., The physics of moving wetting lines J. Colloid $\&$ Interface Sci. 299, (2006) 1-13.

[30] Emady, H.N., Kayrak-Talay, D. \& Lister, J.D., A regime map for granule formation by drop impact on powder beds AIChE J. 59(1), (2013) 96-107.

[31] Ng, W.K., Shen, S.C. \& Tan, R.B.H., Binderless fluidized bed granulation of a type $\mathrm{C}$ hygroscopic pharmaceutical powder with prior humidification treatment Powder Tech. 207, (2011) 9-16.

[32] Kwek, J.W., Lim, M.W., Shen, S.C., Ng, W.K. \& Tan, R.B.H., A pilot scale study on the fluidized bed binderless granulation of a humidified type C hygroscopic pharmaceutical material Powder Tech. 219, (2012) 65-71. 

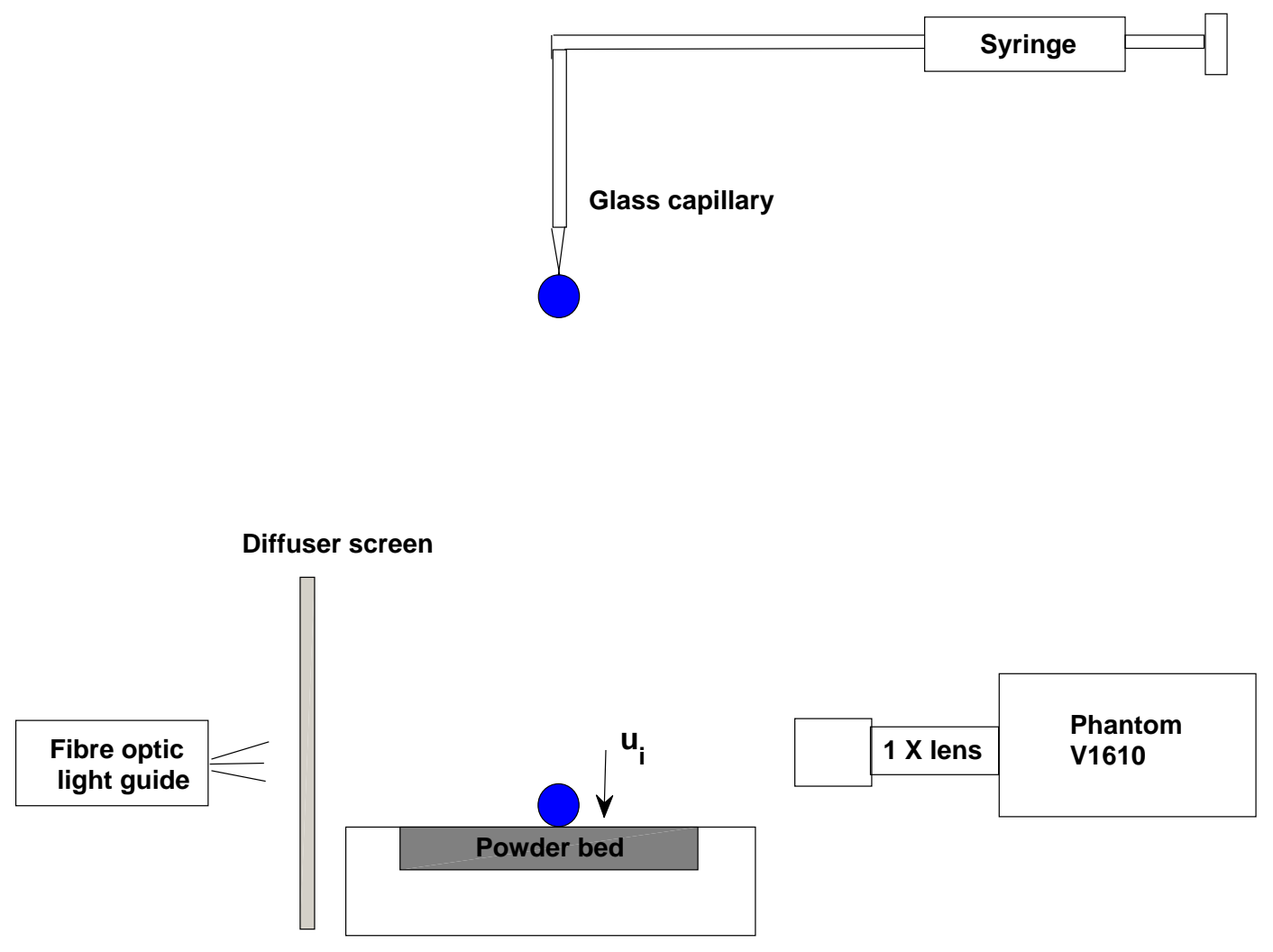

Fig. 1. Schematic of the experimental setup used. 


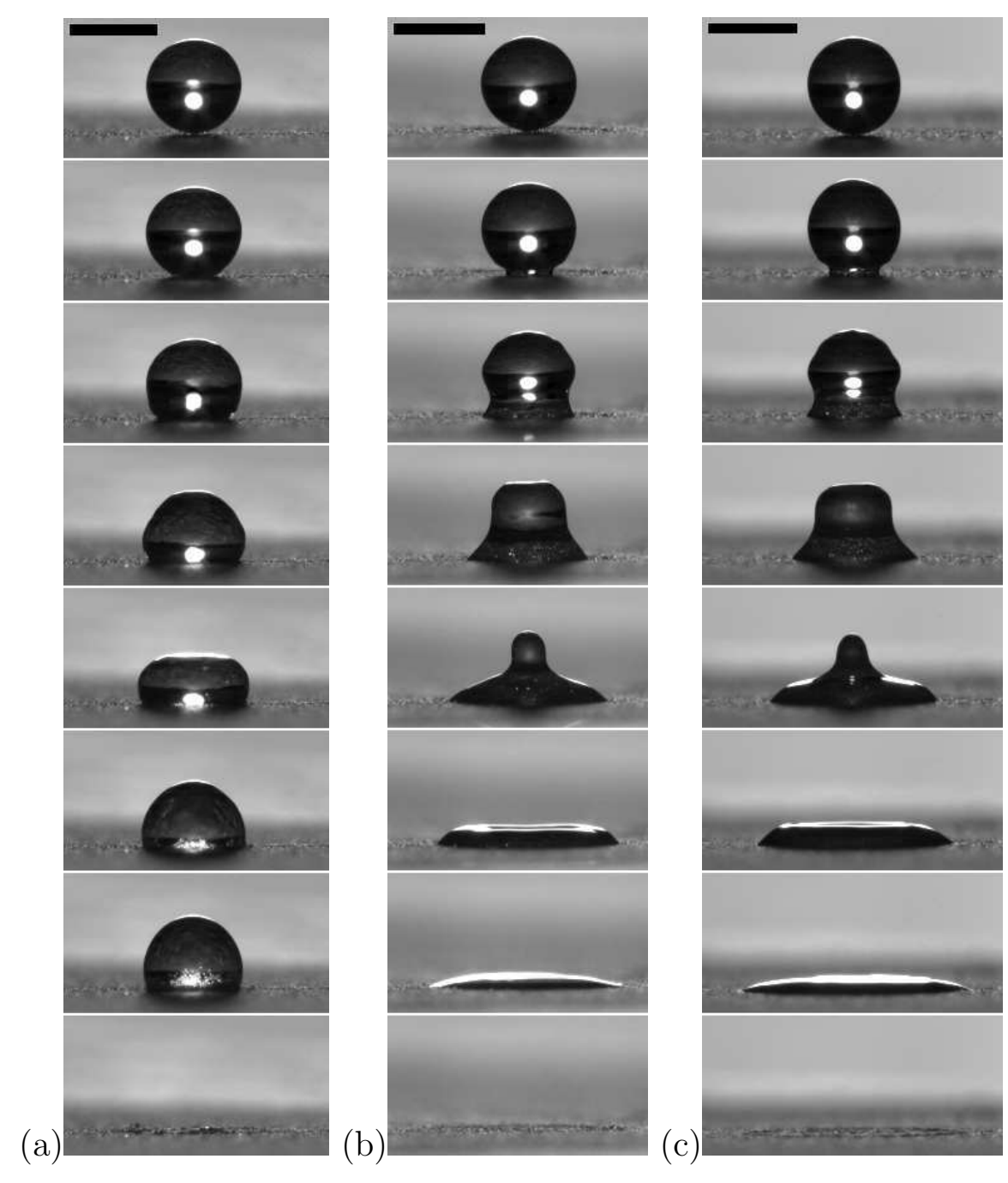

Fig. 2. Water drop impacting onto (a) dry and (b),(c) partially saturated powder with low impact speed. $u_{i}=0.15 \mathrm{~m} / \mathrm{s}, D_{0}=2.1 \mathrm{~mm}$. Moisture contents are $M=0$, 2.5 and $25 \%$ respectively. Frames shown are taken at (a) $t=0,0.5,1.5,2.5,4.5$, $6.5,9.5$, and $211 \mathrm{~ms}$ (b) $t=0,0.5,1.5,2.5,4.5,6.5,9.5$, and $34 \mathrm{~ms}$ (c) $t=0,0.5$, 1.5, 2.5, 4.5, 6.5, 9.5 and $128 \mathrm{~ms}$ and from impact. Scale bars are all $2 \mathrm{~mm}$. See also supplemental video 1 . 


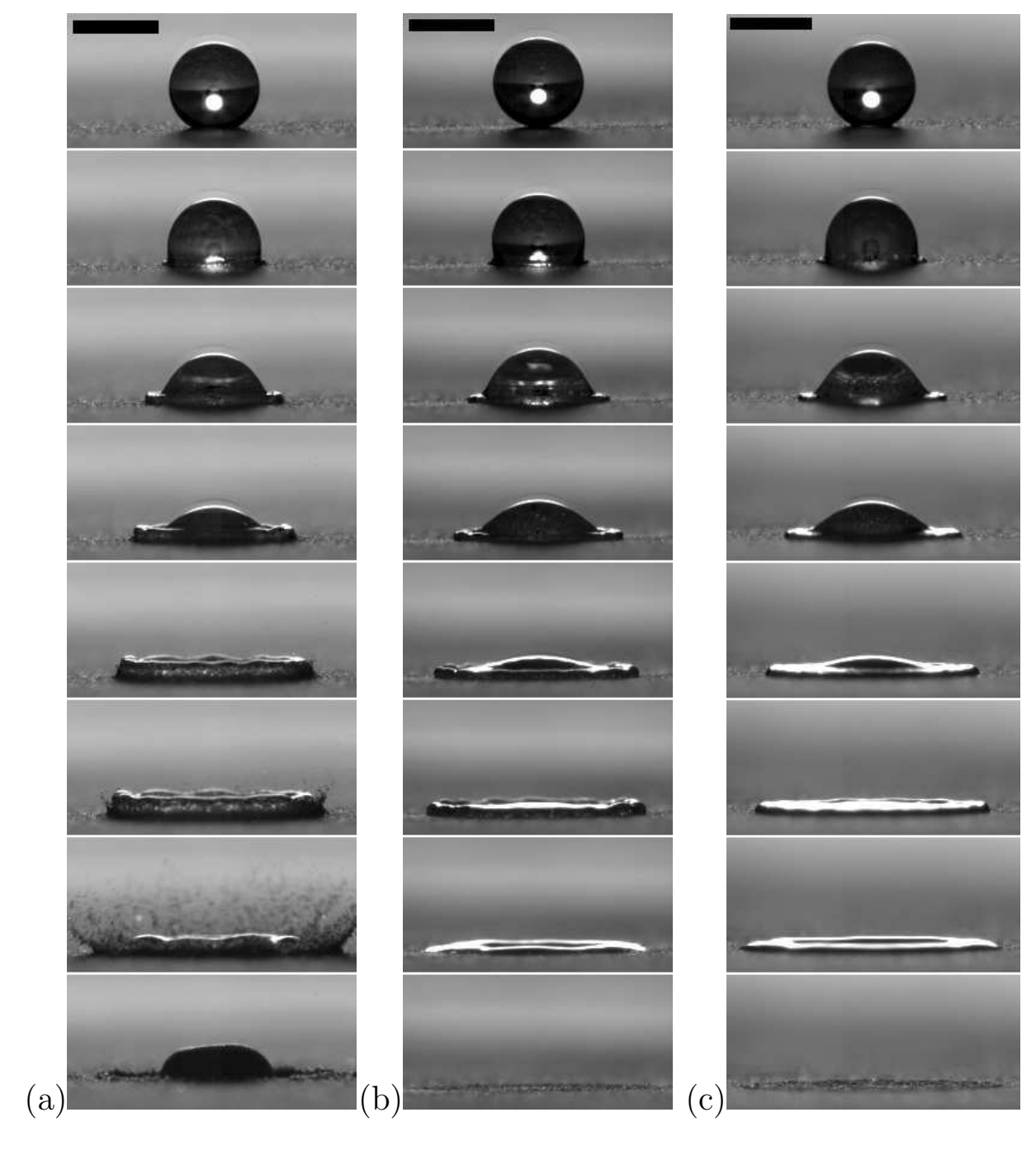

Fig. 3. Water drop impacting onto (a) dry and (b),(c) partially saturated powder with impact speed $u_{i}=2.2 \mathrm{~m} / \mathrm{s}, D_{0}=2.1 \mathrm{~mm}$. Moisture contents are $M=0,2.5$ and $25 \%$ respectively. Frames shown are taken at (b) $t=0,0.2,0.4,0.6,1.0,1.4$, 3.6 , and $190 \mathrm{~ms}(\mathrm{~b}) t=0,0.2,0.4,0.6,1.0,1.4,3.6$, and $13.7 \mathrm{~ms}$ and (c) $t=0,0.2$, $0.4,0.6,1.0,1.4,3.6$, and $41.7 \mathrm{~ms}$ from impact. Scale bars are all $2 \mathrm{~mm}$. See also supplemental video 2 . 
(a)

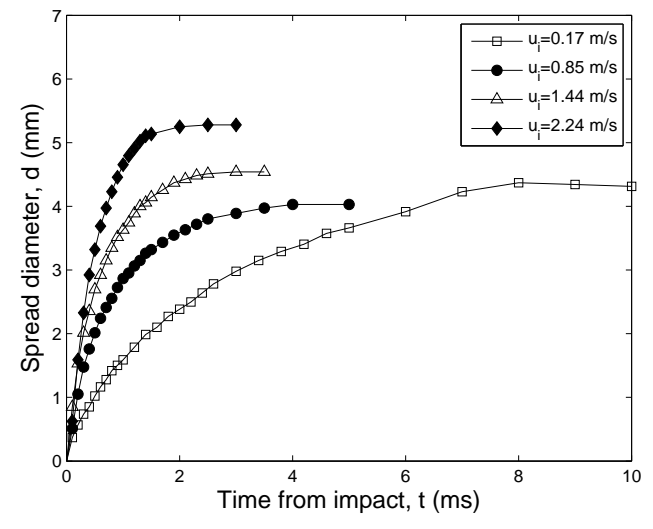

(c)

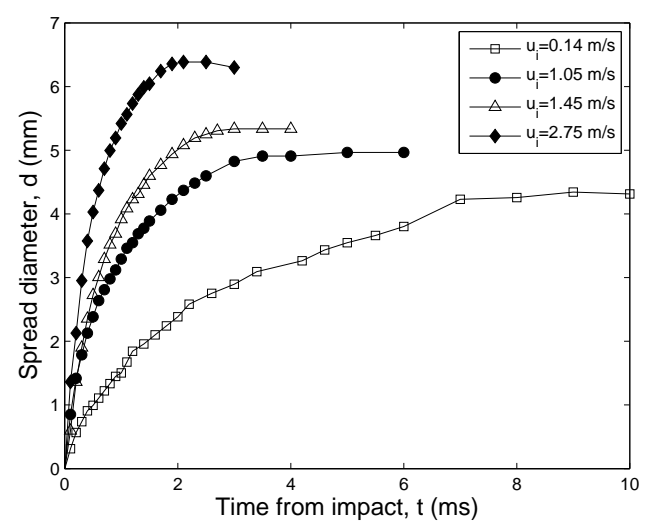

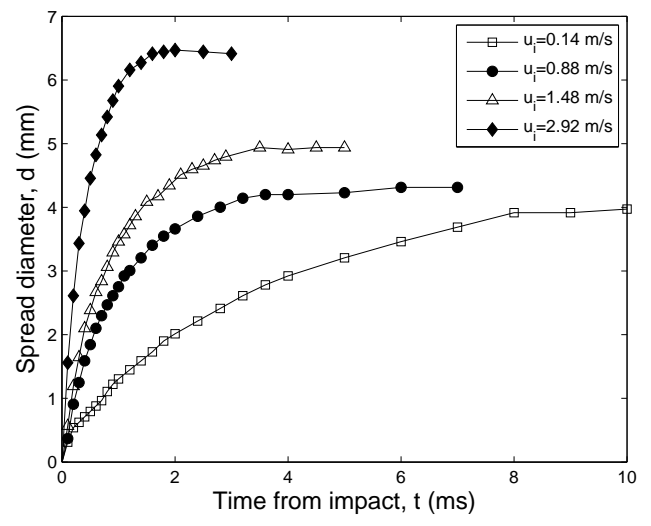

(b)

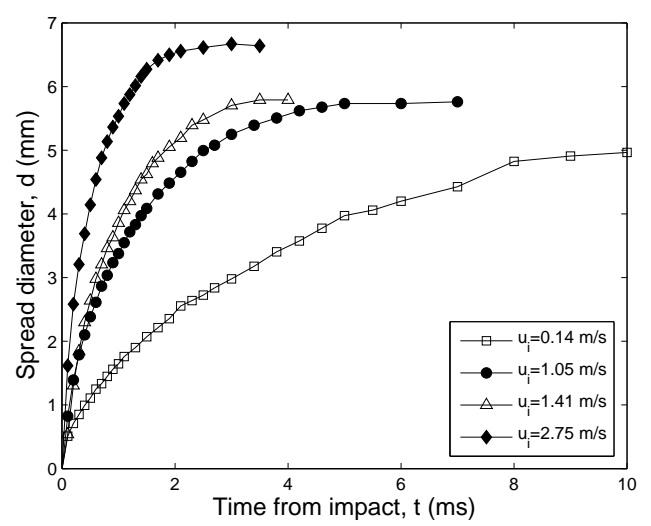

Fig. 4. Spread diameter versus time from impact for increasing impact speed with moisture contents (a) $M=2.5 \%$, (b) $M=5 \%$, (c) $M=10 \%$ and (d) $M=20 \%$. 


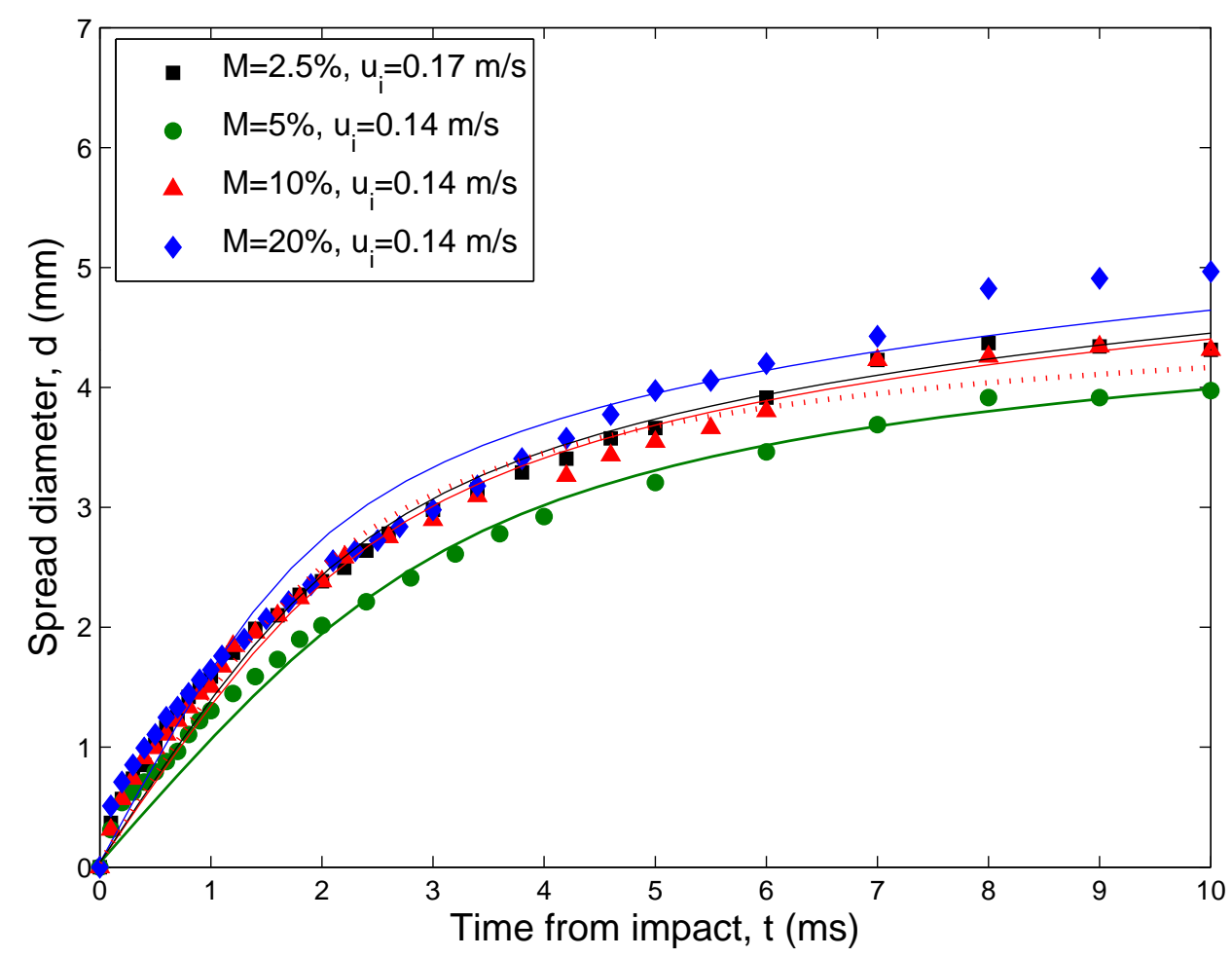

Fig. 5. Comparison between experimental spread diameters and prediction from the model of Clarke et al. (2002) for low-impact speed data. See main text for full details of the model and fitting parameters used. 


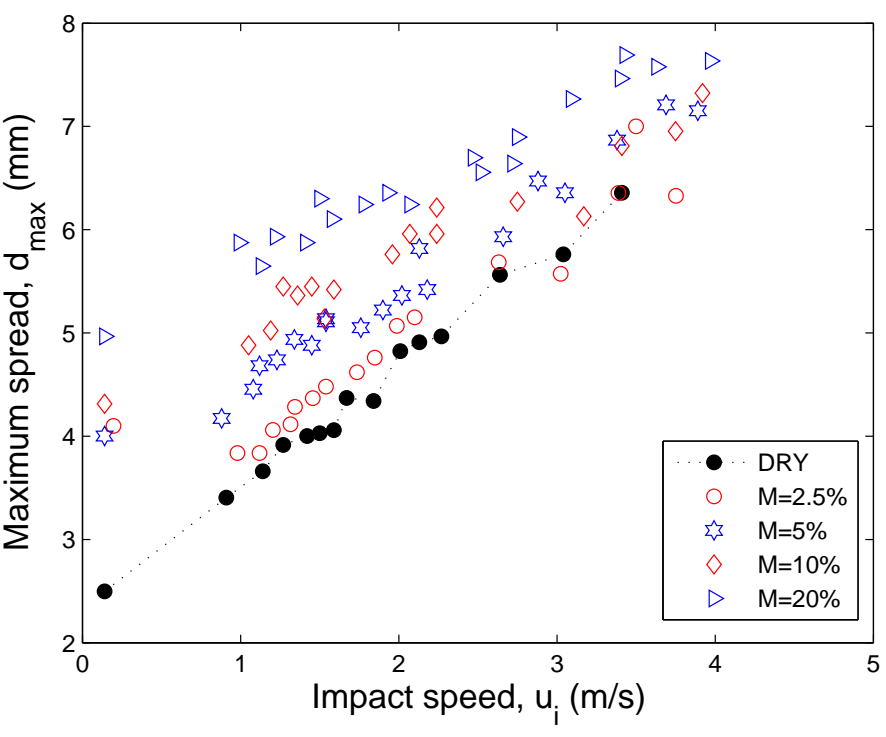

(a)

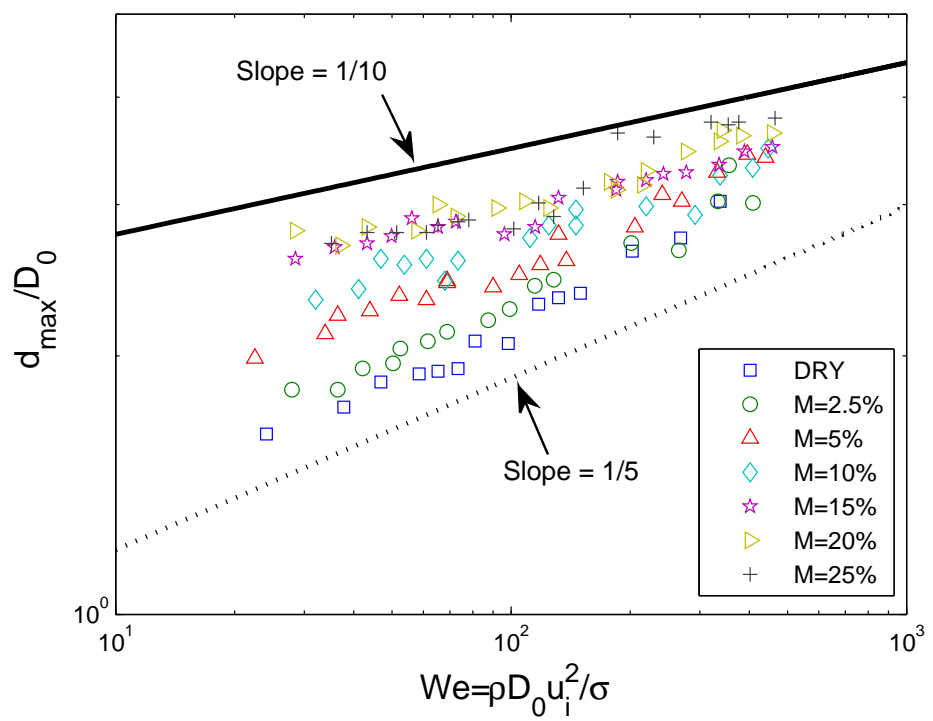

(b)

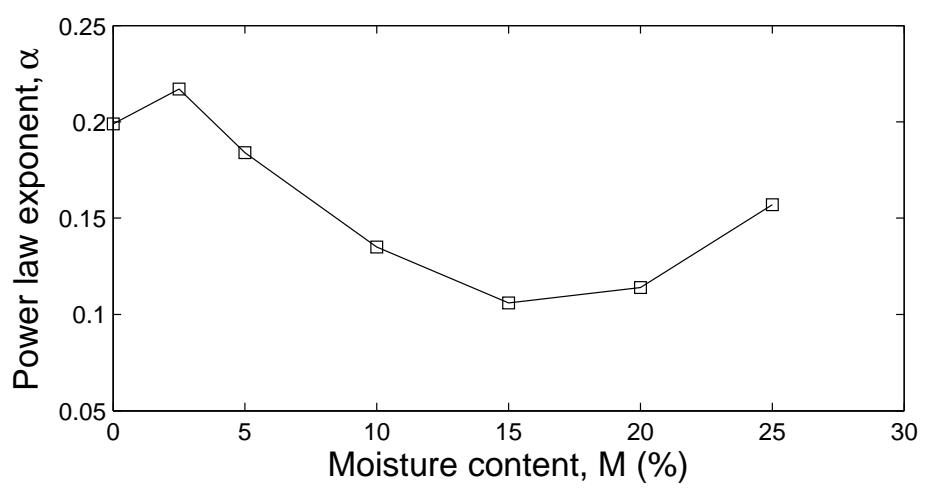

Fig. 6. (a) Maximum spread diameter versus impact speed for dry and pre-wetted powders. (b) Normalised data from (a) plotted as $d_{\max } / D_{0}$ versus the impact Weber number, $W e=\rho D_{0} u_{i}^{2} / \sigma$. (c) Power law exponents derived from fitting $d_{\max } / D_{0} \sim W e^{\alpha}$. 

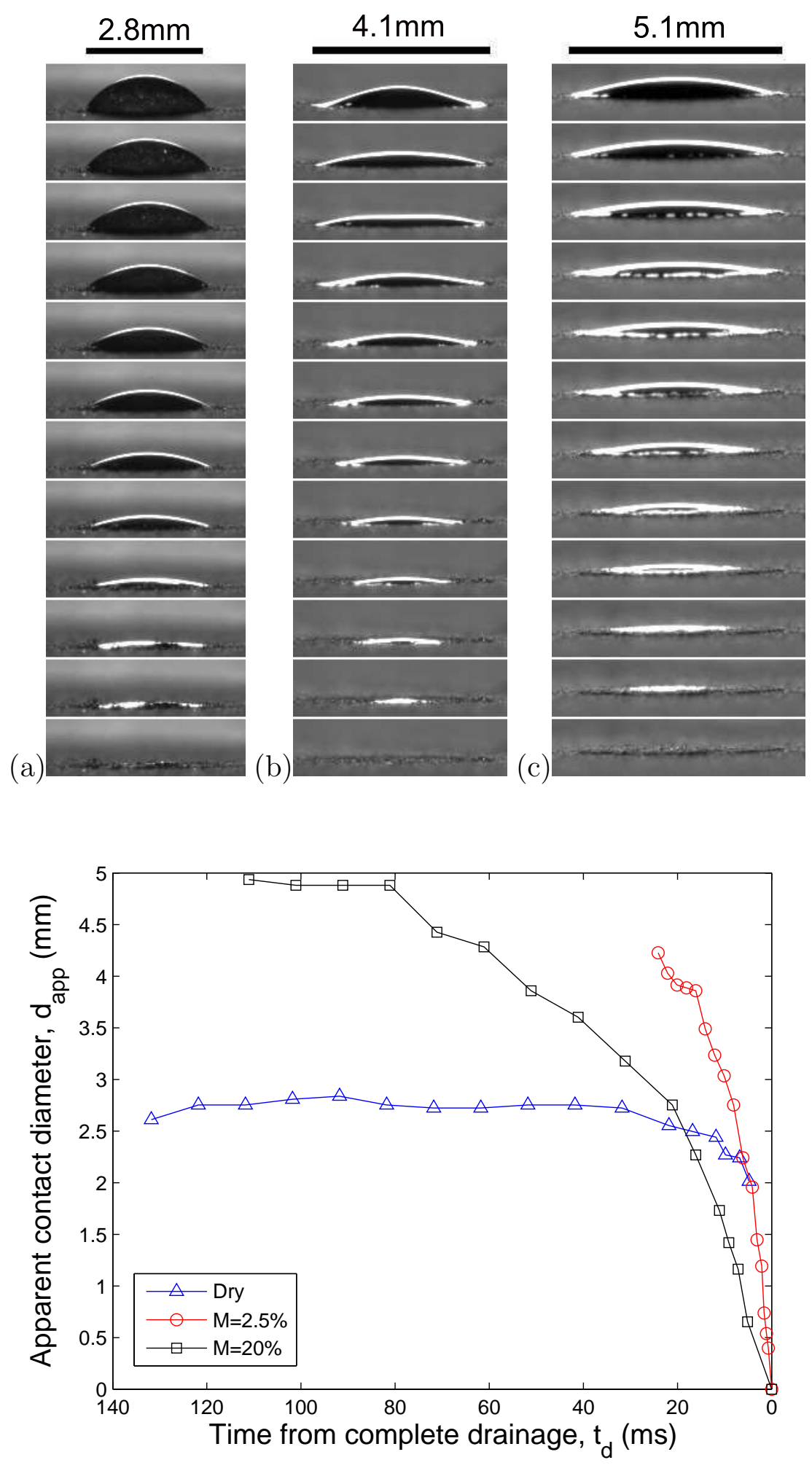

Fig. 7. Drainage of liquid drops into pre-wetted powder. $D_{0}=2.1 \mathrm{~mm}, u_{i}=0.14$ $\mathrm{m} / \mathrm{s}$, with (a) $M=0 \%$, (b) $M=2.5 \%$ and (c) $M=20 \%$. The first frame is taken after the drop reaches maximum deformation $\left(d_{\max }\right)$ with (a) $t_{0}=80 \mathrm{~ms}$, (b) $t_{0}=10 \mathrm{~ms}$ and (c) $t_{0}=16 \mathrm{~ms}$ and the interval between subsequent frames is (a) $d t=10 \mathrm{~ms}$, (b) $d t=2 \mathrm{~ms}$ and (c) $d t=10 \mathrm{~ms}$. (d) Apparent contact diameter versus time from complete drainage for the realisations shown in (a), (b) and (c) respectively. 


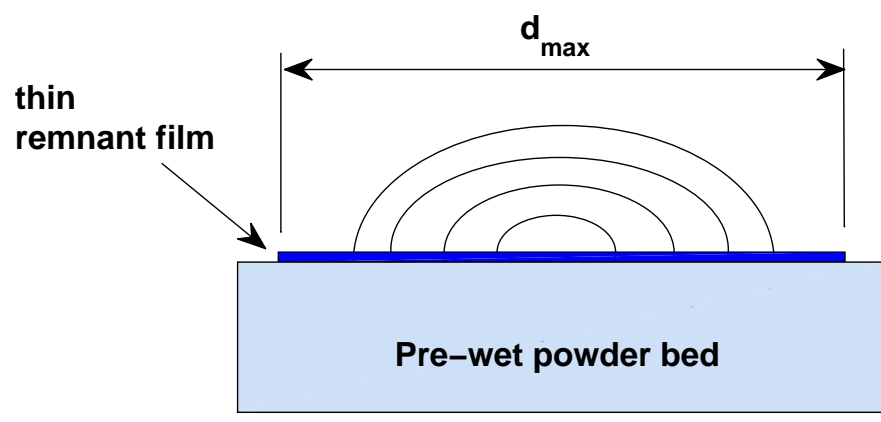

(a)

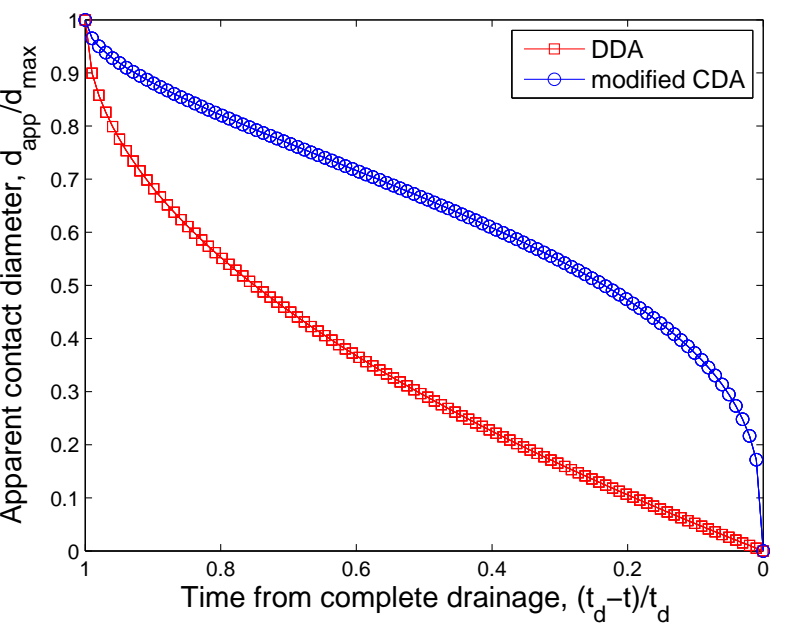

Fig. 8. (a) Schematic of the modified CDA-type drainage process for pre-wet powder beds, showing a thin remanant film with constant diameter $d_{\max }$ and decreasing apparent contact radius. (b) Predicted apparent contact radius versus time for modified CDA and DDA-type drainage from equations (5) and (6) respectively. 


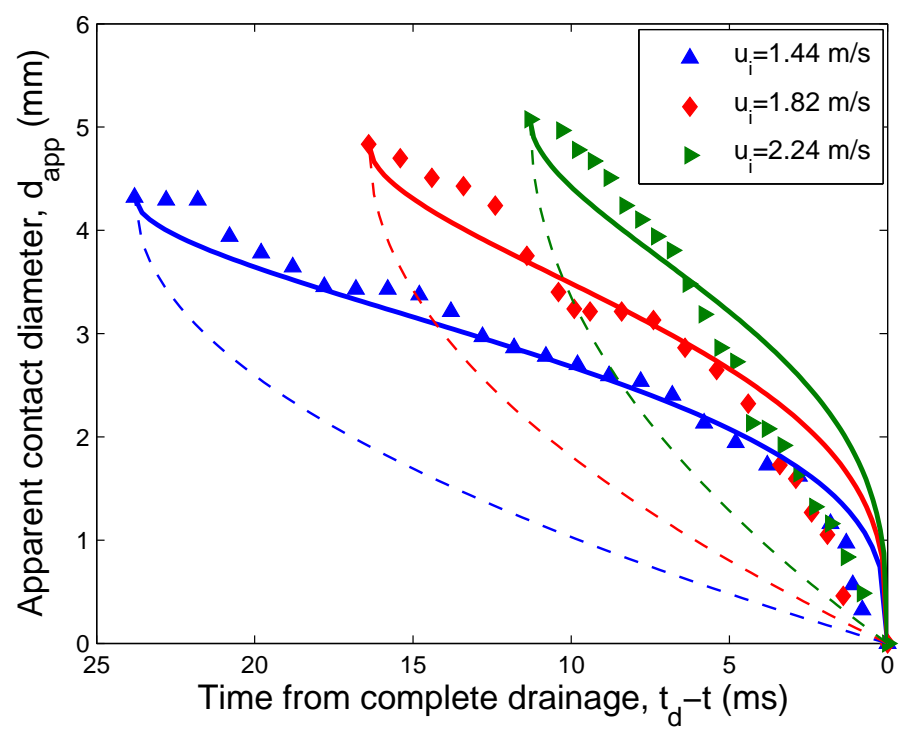

Fig. 9. Apparent contact diameter versus time for pre-wetted powder for different drop impact speeds $(M=2.5 \%)$. The discrete data points correspond to the experimental data, whilst the solid and dashed lines correspond to the predicted evolution from equations (4) and (5) respectively. 
(a)

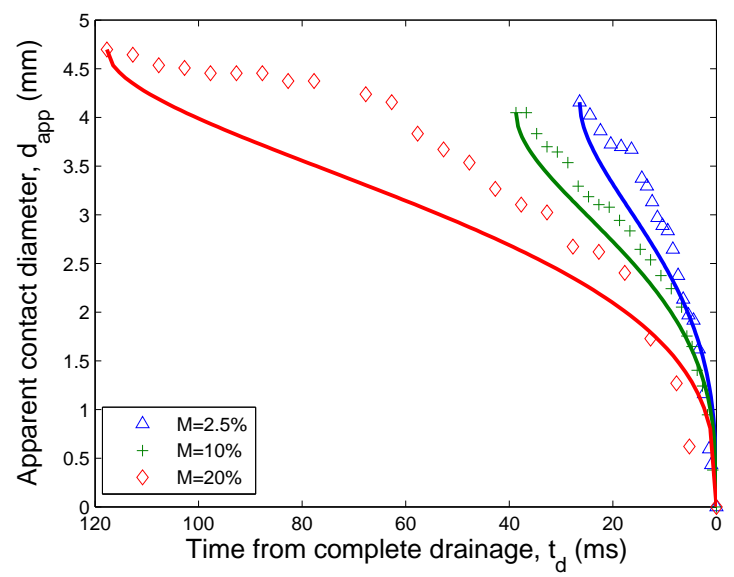

(c)

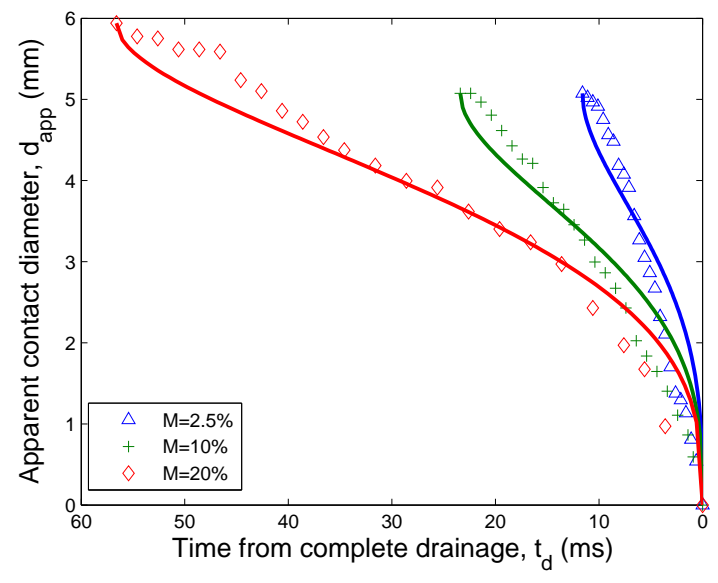

(b)

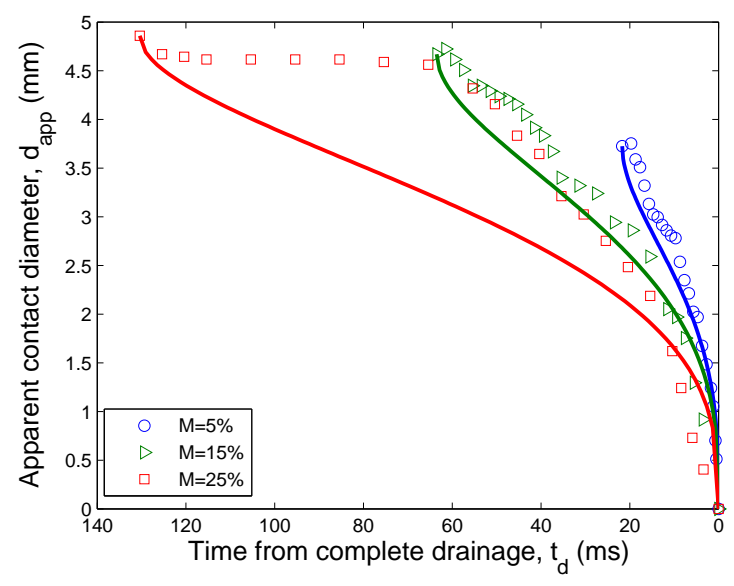

(d)

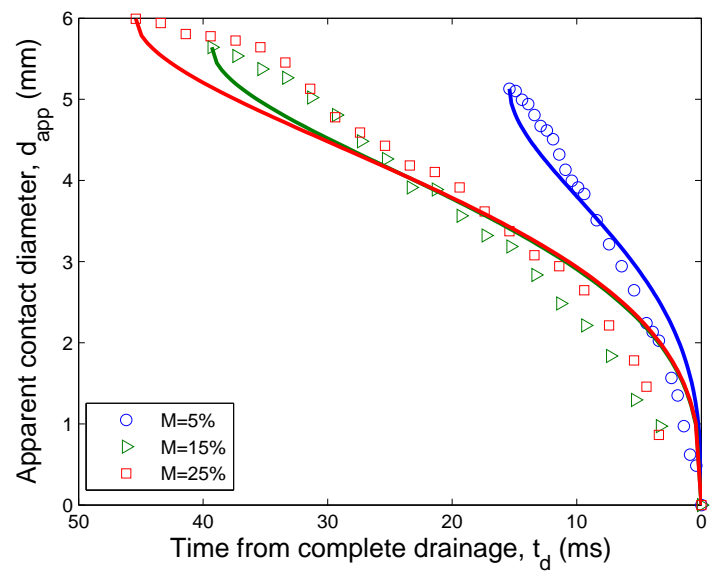

Fig. 10. Apparent contact diameter versus time for pre-wetted powders with (a,c) $M=2.5,10$ and $20 \%$ and (b,d) $M=5,15$ and $25 \%$. In both (a) and (b), the drops were released from just above the powder surface with $u_{i} \approx 0.15 \mathrm{~m} / \mathrm{s}$, whilst in both $(\mathrm{c})$ and $(\mathrm{d})$ the impact speed $u_{i} \approx 2 \mathrm{~m} / \mathrm{s}$. The solid lines correspond to the predicted evolution from equation (4). 


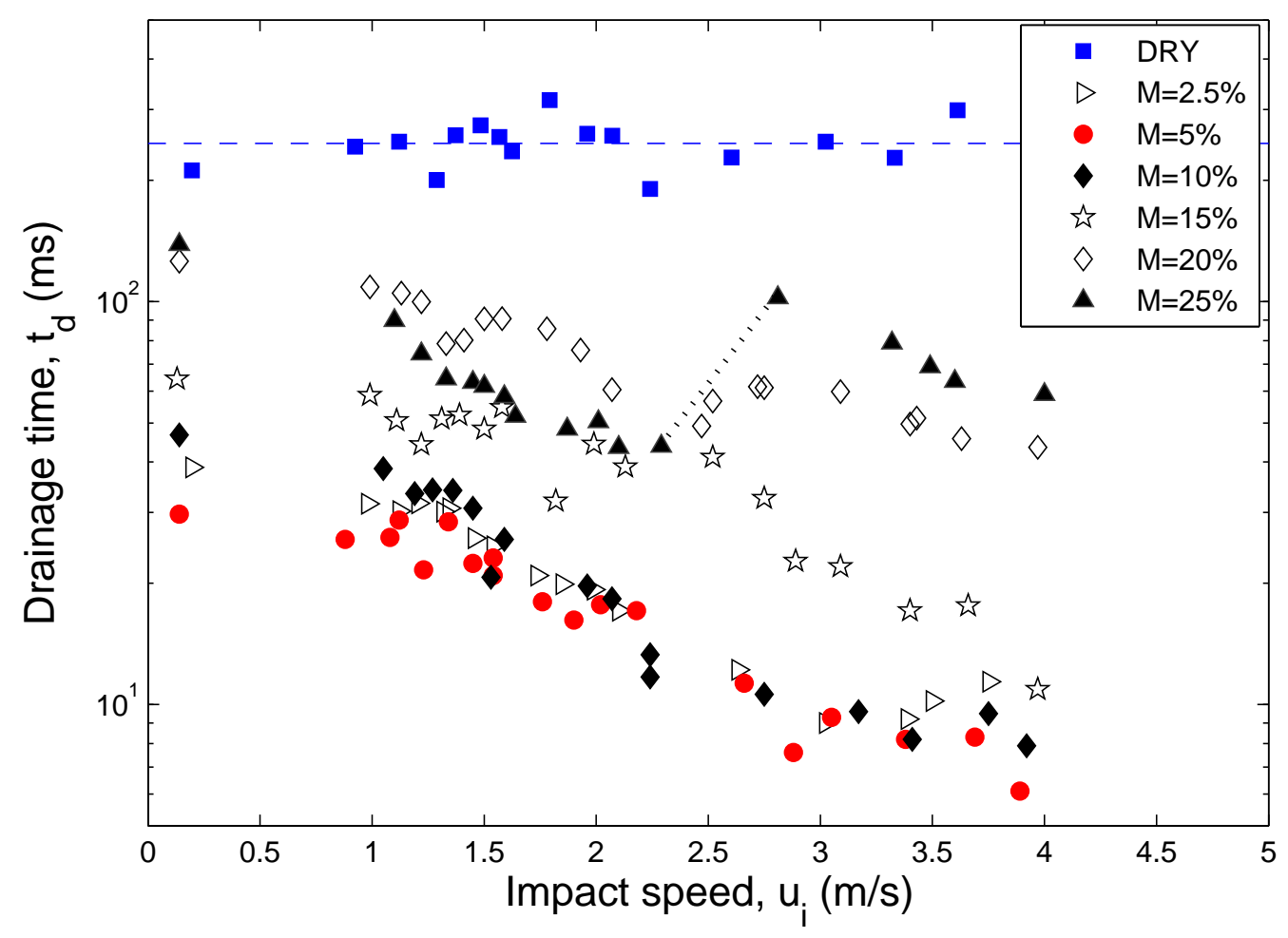

Fig. 11. Total drainage time versus drop impact speed for dry and pre-wetted powders. The dashed blue line represents the mean time of $247 \mathrm{~ms}$ for the dry powder, whilst the fastest penetration $(M=5 \%)$ are highlighted in red. The dotted black line shows the increase in $t_{d}$, which occurs for $M=25 \%$ for $u_{i}>2.5 \mathrm{~m} / \mathrm{s}$. 


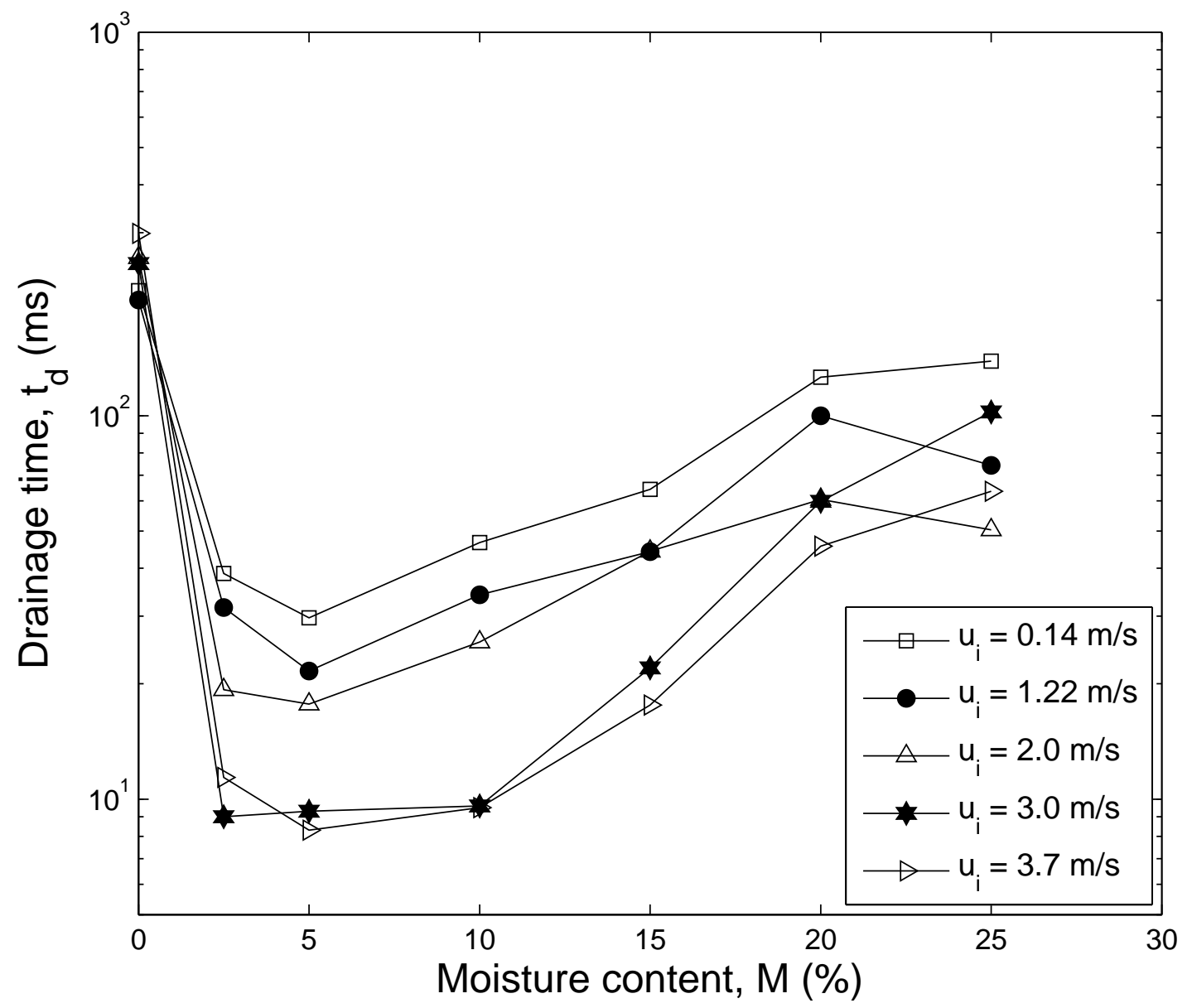

Fig. 12. Total drainage time versus moisture content for select impact speeds $u_{i}=0.14,1.22,2.0,3.0$ and $3.7 \mathrm{~m} / \mathrm{s}$. 



\section{RECENT REPORTS}

12/94 The Mechanics of a Chain or Ring of Spherical Magnets

Hall

Vella

Goriely

12/95 On-Lattice Agent-based Simulation of Populations of Cells within the Open-Source Chaste Framework

Figueredo

Joshi

Osborne

Byrne

Owen

12/96 Mathematical Biomedicine and Modeling Avascular Tumor

Byrne Growth

12/97 Inference of the genetic network regulating lateral root initiation in Arabidopsis thaliana

Muraro

Voß

Wilson

Bennett

Byrne

De Smet

Hodgman

King

12/98 Axisymmetric bifurcations of thick spherical shells under inflation and compression

deBotton

Bustamante

Dorfmann

12/99 Calculus from the past: Multiple Delay Systems arising in Cancer

Wake

Cell Modelling

Byrne

12/100 Nonlocal models of electrical propagation in cardiac tissue: electrotonic effects and the modulated dispersion of repolarization

Bueno-Orovio

Kay

Grau

Rodriguez

Burrage

12/101 Microfluidic Immunomagnetic Multi-Target Sorting A Model for Controlling Deflection of Paramagnetic Beads

Tsai

Griffiths

Stone

12/102 A New Lattice Boltzmann Equation to Simulate Density-Driven Convection of Carbon Dioxide

Allen

Reis

Sun

12/103 Control and optimization of solute transport in a porous tube

Griffiths

Howell

Shipley

12/104 Air-cushioning in impact problems

Moore

Ockendon

Oliver

12/105 Strain controlled biaxial stretch: An experimental characterization

Pancheri of natural rubber

Dorfmann

12/106 Non-linear modeling of active biohybrid materials

Paetsch

Dorfmann

12/107 Coalescence of Liquid Drops: Different Models Versus Experi- $\quad$ Sprittles 
12/108 Adjoint Based A Posteriori Analysis of Multiscale Mortar Discretizations with Multinumerics

Tavener

Wildey

12/109 Dynamics of mechanically induced fiber reorientation in the ma-

Melnik

terial reinforced by two families of fibers

Goriely

12/110 Multiscale stochastic reaction-diffusion modelling: application to actin dynamics in filopodia

Erban

Flegg

Papoian

12/111 Exploiting the Synergy Between Carboplatin and ABT-737 in the Treatment of Ovarian Carcinomas

Jain

Richardson

Meyer-Hermann

Byrne

12/112 The integration of hormonal signaling networks and mobile mi-

Muraro croRNAs is required for vascular patterning in Arabidopsis roots

Pound

Help

Lucas

Chopard

Byrne

Godin

Hodgman

King

Pridmore

Helariutta

Bennett

Bishopp

12/113 Fast solution of Cahn-Hilliard Variational Inequalities using Im-

Bosch plicit Time Discretization and Finite Elements

Stoll

Benner

12/114 An Embedding Technique for the Solution of Reaction-Diffusion Equations on Algebraic Surfaces with Isolated Singularities

Rockstroh

März

Ruuth

12/115 Mathematicians at the Movies: Sherlock Holmes vs. Professor

Moulton Moriarty

Goriely

13/01 Rotation, inversion, and perversion in anisotropic elastic cylindri-

Goriely cal tubes and membranes

Tabor

Copies of these, and any other OCCAM reports can be obtained from:

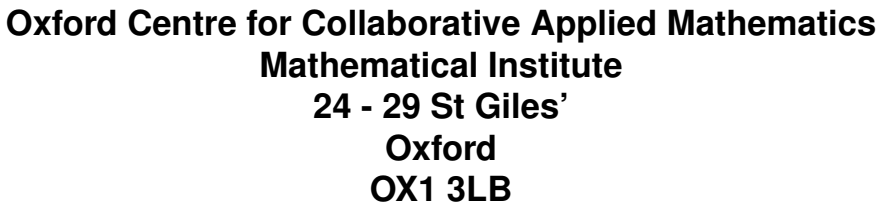


England

www.maths.ox.ac.uk/occam 